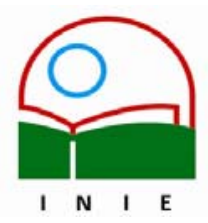

Universidad de Costa Rica

Facultad de Educación

Instituto de Investigación en Educación

ACTUALIDADES INVESTIGATIVAS EN EDUCACION

\title{
LAS ESTRATEGIAS DIDÁCTICAS EN LA FORMACIÓN DE DOCENTES DE EDUCACIÓN PRIMARIA
}

\section{Wilfredo Gonzaga Martínez ${ }^{1}$}

\begin{abstract}
Resumen: El artículo tiene como propósito dar a conocer los resultados de un estudio sobre el proceso didáctico y la incorporación de estrategias innovadoras en los procesos de enseñanza y aprendizaje que se llevan a cabo en el desarrollo de los cursos que conforman el área pedagógica del plan de estudio, para la formación de docentes de Educación Primaria, en la Sede de Occidente de la Universidad de Costa Rica.

Los cursos que se consideraron fueron: Introducción a la pedagogía, Didáctica General, Principios de Curriculum, Investigación Educativa, Psicología Educativa, Ciencias en la Educación Primaria, Práctica Docente y Evaluación de los Aprendizajes.

La investigación se orientó con el siguiente problema general: ¿Cuáles son las estrategias didácticas en la enseñanza del área pedagógica, aplicadas en la formación inicial de docentes para la Educación Primaria en la Sede de Occidente de la Universidad de Costa Rica?

En el estudio se analizaron y se confrontaron los programas de los cursos mencionados, considerando las estrategias didácticas que se aplican para su desarrollo, según el criterio de docentes y alumnos. En este análisis se describe la coherencia entre estos dos aspectos, las tendencias pedagógicas que subyacen en las estrategias didácticas y las innovaciones que se desarrollan como parte de los procesos de enseñanza y aprendizaje.

El proceso investigativo demuestra que el plan de estudio considera como referencia pedagógica un enfoque humanista; mientras que los programas de los cursos y la práctica docente no logran desligarse de la didáctica tradicional, con tendencia a incorporar aspectos del Constructivismo y de la Pedagogía Crítica. En el estudio no se logró apreciar la incorporación de estrategias didácticas innovadoras de manera significativa.
\end{abstract}

\section{Palabras clave: FORMACIÓN DE DOCENTES/ ENSEÑANZA PRIMARIA/ ESTRATEGIAS DIDACTICAS/}

\begin{abstract}
This article is intended to present the results of a study on the didactic process and the incorporation of didactic strategies in the context of pedagogy, and its applications for the initial training of primary school teachers in the UCR's Western Site.

The courses that were considered were: Introduction to Pedagogy, General Didactics, Principles of Study Plans, Research on Education, Psychology on Education, Sciences in Primary School, Teaching Practice, Educational and Learning Evaluation.

The investigation was oriented to the following general problem: What are the didactic strategies for training in the context of pedagogy, applied to the initial training of teachers in the UCR's Western Site?

In the study, the programs of the aforementioned courses were analyzed and subject a review, considering the didactic strategies that are being applied for their development, in accordance with the points of view of teachers and students. In this analysis the pedagogical coherence between these two aspects is analyzed, the tendencies that lie below the didactic strategies and the innovations that are developed as part of the teaching and learning processes.

The study demonstrates that the study plan considers a humanist approach as a pedagogical reference; whereas the programs of the courses and the teaching practice do not manage to be separated from the traditional didactics, tending to include aspects of constructivism and critical pedagogy theoretic. The study was not able to appraise any significant incorporation of innovating didactic strategies.
\end{abstract}

Keywords: TEACHER TRAINING/ PRIMARY EDUCATION/TEACHING STRATEGIES/

\begin{abstract}
${ }^{1}$ Magister en Evaluación Educativa, Licenciatura y Bachillerato en Ciencias de la Educación Primaria, todos títulos de la Universidad de Costa Rica. Actualmente es profesor de la Escuela de Formación Docente e Investigador del Instituto de Investigación en Educación.
\end{abstract}

Correo electrónico: wgonzaga04@yahoo.es

Artículo recibido: 28 de abril, 2005

Aprobado: 29 de junio, 2005 


\section{Introducción}

El presente artículo se refiere a la investigación "Estrategias didácticas empleadas en la enseñanza del área pedagógica en el plan de formación inicial de docentes para la Educación Primaria, en la Sede de Occidente de la Universidad de Costa Rica"; la cual se enmarca en el proyecto de investigación "Estrategias didácticas empleadas en la enseñanza del área pedagógica en los planes de formación inicial de docentes para la Educación Primaria, en las universidades estatales costarricenses", el cual forma parte del proyecto que ha venido desarrollando la Coordinación Educativa y Cultural Centroamericana (CECC): "Apoyo al mejoramiento de la formación inicial de docentes de la Educación Primaria o Básica".

La Educación Primaria es fundamental en el sistema educativo costarricense y se espera que sea aprobado por el $100 \%$ de los estudiantes. La expectativa es además que, en este nivel de formación, se fomente el logro de una serie de procesos formativos como: socialización, desarrollo integral, reconocimiento y desarrollo de aptitudes y valores.

La calidad de la Educación Primaria depende de múltiples factores, uno de los más importantes es la formación de docentes. Por lo que se requiere contar con un docente creativo, que posea un conocimiento amplio y profundo de lo qué, cómo y cuándo debe enseñar; con un manejo apropiado de estrategias de enseñanza y aprendizaje, de procedimientos e instrumentos de evaluación, y con una clara comprensión de lo que significa su trabajo de aula, tanto en el ámbito del desarrollo individual y grupal de los estudiantes, como del impacto social de la labor educativa, capaz de vencer limitaciones y obstáculos y de llevar a cabo una práctica docente que satisfaga las expectativas del sistema educativo y de la sociedad en general. Por esta razón, el proceso de formación de docentes es tan importante que se le brinda la atención y reflexión que merece.

Uno de los aspectos básicos de la preparación de docentes lo constituye el proceso mismo de formación, en el cual la aplicación de determinadas estrategias didácticas contribuye a construir y apropiarse de formas de trabajo que posteriormente, sirven de referencia a los docentes para organizar su propia práctica pedagógica, al constituirse, estas estrategias en "modelos" que tienden a ser reproducidos.

En este estudio se indagó la formación de los docentes de Educación Primaria, desde las estrategias didácticas aplicadas en el desarrollo de los cursos del área pedagógica, al considerar que esta área es esencial en la construcción del marco conceptual y de acción, que fundamenta la práctica educativa. 
Se identificaron formas en que el curriculum se desarrolla, privilegiando el abordaje en dos dimensiones: lo previsto para su ejecución en los planes y programas de estudio, y la manera en que éstos son llevados a la práctica.

Los resultados generados por la investigación pueden incidir en el mejoramiento del diseño y ejecución de estos planes de estudio, así como en la organización de los programas de capacitación, y en el trabajo pedagógico de los maestros de Educación Primaria.

\section{Problema o interrogante general}

¿Cuáles son las estrategias didácticas del área pedagógica, aplicadas en la formación inicial de docentes para la Educación Primaria, en la Sede de Occidente de la Universidad de Costa Rica?

\section{Subproblemas o interrogantes específicos}

1. ¿Cuáles son las estrategias didácticas presentes, en el plan de estudio y los programas de los cursos del área pedagógica: Pedagogía, Curriculum, Didáctica General, Didácticas Específicas, Materiales Educativas, Psicología Educativa, Investigación Educativa, Práctica Docente, Tecnología Educativa y Evaluación de los Aprendizajes?

2. ¿Cuáles estrategias didácticas se utilizan en el desarrollo de los cursos del área pedagógica: Pedagogía, Curriculum, Didáctica General, Didácticas Específicas, Materiales Educativos, Psicología Educativa Investigación Educativa, Práctica Docente, Tecnología Educativa y Evaluación de los Aprendizajes?

3. ¿Existe vinculación entre las estrategias didácticas previstas en los programas de cursos, las tendencias pedagógicas y las características generales de los planes de estudio?

4. ¿Existe vinculación entre las estrategias didácticas previstas en los programas de cursos y las estrategias didácticas empleadas en el desarrollo de los mismos del área pedagógica?

5. ¿Cuáles de los cursos estudiados incorporan estrategias didácticas innovadoras ${ }^{2}$ tanto en los programas como en su práctica real? En caso de presentarse esta situación ¿en qué consisten estas estrategias innovadoras?

\footnotetext{
2 Para efectos de la presente investigación se entenderá por estrategias didácticas innovadoras, aquellas vinculadas con la tecnología e informática (multimedia, Internet, textos electrónicos, etc.). 


\section{Fundamentación teórica}

La formación de docentes ha sido un tema de mucho interés, en los últimos años, lo anterior se evidencia en las diferentes publicaciones y actividades académicas (reuniones técnicas, seminarios, congresos), que se han organizado con el propósito de analizar la situación y los problemas relevantes que presenta, así como la elaboración de propuestas de mejoramiento para la formación de los docentes de la Educación Primaria o Básica.

En cuanto a las recomendaciones que se han originado en esas actividades, conviene señalar, las propuestas encomendadas en el Seminario Regional, para la Formación Docente Inicial (UNESCO y UPEL, 1996), que a continuación se indican:

- La formación docente inicial debe enmarcarse en un proceso de formación permanente.

- La investigación debe ser uno de los ejes de los programas de formación docente inicial.

- En el desarrollo de los programas de formación docente inicial se debe partir de las necesidades del sistema educativo; considerar la formación humanística; promover una formación teórico práctica, en contacto con la realidad; incorporar una práctica docente temprana, favorecer la formación integral, personal y la autoestima en los futuros docentes, estimular la producción de textos; estimular el autoaprendizaje y la formación permanente; darle especial atención a la formación de valores.

- Las políticas y programas de formación de los Ministerios de Educación, deben sustentarse en la investigación.

- Establecer programas conjuntos entre los centros formadores de docentes y las instituciones educativas.

- Evaluar, integralmente, los sistemas educativos, para su mejoramiento y no como un mecanismo de los procesos de ajuste estructural que se dan en la región.

Las anteriores recomendaciones mantienen su vigencia como lineamientos orientadores de los procesos de formación de docentes y deberían, en alguna medida, reflejarse en las propuestas de los planes de formación de las instituciones que se encargan de la educación de los docentes.

La Coordinación Educativa y Cultural Centroamericana (CECC) ha venido impulsando distintos proyectos dirigidos al estudio y mejoramiento de la Educación Primaria. En septiembre de 1996, la CECC, organizó en Tegucigalpa, Honduras, un Taller Regional de formación inicial de docentes de Educación Primaria o Básica. Entre las conclusiones generales que se obtuvieron en este taller se destacan las siguientes:

- La formación inicial de docentes para la Educación Primaria o Básica presenta diferentes niveles de desarrollo. En Costa Rica, se ha consolidado como una carrera post- 
secundaria y universitaria desde 1936; en El Salvador, se inicia dicha consolidación; en Panamá, se tiene un sistema mixto en vías de cambio; mientras en los otros países, se sigue formando al maestro en el nivel secundario.

- Los planes de estudio para la formación inicial de docentes son similares en los contenidos generales; pero difieren en: a) la fundamentación pedagógica, b) en las metodologías que se plantean para la puesta en marcha de los procesos de enseñanzaaprendizaje y c) en la profundidad del tratamiento de los contenidos.

- Las políticas nacionales de desarrollo general y de desarrollo educativo en particular impactan la formación inicial de docentes.

- No existe en el ámbito centroamericano, en general, políticas de formación de docentes que se traduzcan en acciones formativas claras en los campos de la educación sobre los derechos humanos; el empleo de las computadoras y el "software" educativo; la atención de la diversidad cultural y étnica; el empleo racional, la conservación y el respeto por los recursos naturales, y de la promoción del desarrollo comunitario.

- En general, no se explicita la existencia de sesgos sexistas en la formación de docentes, ni en la legislación vigente o en el empleo de los docentes. Pero no existe evidencia de que se esté preparando a los docentes para enfrentar una educación sin discriminación por sexo. En consecuencia este tema demanda un estudio amplio por cuanto la mayoría de estudiantes que acceden a esta carrera, en el nivel inicial, son mujeres.

La formación de docentes responde a determinadas tendencias o enfoques pedagógicos. En relación con las tendencias, Esther Carolina Pérez Juárez (Panza, Pérez y Morán, 1997) y Ana Cecilia Hernández Rodríguez (1999) conceptualizan las siguientes tres tendencias pedagógicas presentes en las prácticas educativas actuales: tradicional, tecnocrática y crítica. La caracterización de éstas, de acuerdo con las autoras ya mencionadas se presenta a continuación:

Tendencia pedagógica tradicional: Ésta se centra "en la transmisión de contenidos", predomina el uso de métodos expositivos (se explica, se escribe en la pizarra, se dicta, se entregan fotocopias, etc.) y de actividades que promueven la memorización y la repetición de contenidos. La enseñanza se fundamenta en la "autoridad" (o autoritarismo) del profesor, él posee el conocimiento, toma las decisiones y define las estrategias de enseñanza y de evaluación. Esta se orienta a la comprobación "mecánica" de los aprendizajes, mediante una "devolución" de contenidos "aprendidos".

El conocimiento se toma como un producto acabado que el estudiante debe asimilar, mediante una serie de actividades, pero su posición frente a estos procesos es receptiva. 
La tendencia tecnocrática o tecnológica: Se caracteriza por el uso de diversos medios audiovisuales, multimedia y otros relacionados con la tecnología informática; el rol del profesor y del estudiante podría cambiar un poco, con respecto a la tendencia tradicional. El docente al incorporarse a equipos interdisciplinarios que preparan materiales educativos mejor elaborados y sofisticados; el estudiante porque, podría tener acceso a múltiples medios para su aprendizaje; no obstante la posición epistemológica podría mantenerse similar al enfoque anterior, al concebirse el conocimiento como una verdad dada, objetiva y neutra que hay que asimilar.

La tendencia constructivista: Se puede afirmar que el constructivismo es una posición epistemológica que fundamenta y alimenta perspectivas pedagógicas con diversos énfasis, pero, siempre propiciando la participación activa del sujeto que aprende en la construcción y apropiación del conocimiento.

Es obligado señalar el desarrollo y la contribución que se hace a esta corriente por autores norteamericanos como Ausubel, Bruner y Novak, en la conceptualización del aprendiz, a partir del conocimiento previo y de una disposición afectiva hacia el nuevo aprendizaje, produciéndose así un aprendizaje duradero y con sentido (significativo).

En una posición constructivista, el formador de docentes cumple también una función relevante en su condición de guía y facilitador del proceso. En este sentido, debe poseer actitudes, conocimientos y habilidades que le permitan ser un mediador efectivo entre la cultura sistematizada y el conocimiento del contexto o de los múltiples contextos desde los cuales se ha generado los conocimientos previos de los estudiantes. En cuanto a la responsabilidad de los estudiantes, estos deben desarrollar, por medio de los procesos de enseñanza y aprendizaje (dinámicos y creativos), la motivación requerida para la construcción de nuevos aprendizajes. Parte de esa motivación la proporciona su participación responsable en la definición de objetivos, de contenidos y planificación de experiencias de aprendizaje significativo en relación con su propio contexto.

La tendencia crítica: En esta posición el contexto, como un todo, se constituye en el espacio en el cual el conocimiento adquiere significado. La educación no se puede entender al margen de un contexto y de la sociedad; una educación desarticulada de su contexto carece de valor.

La pedagogía crítica se enfrenta a los problemas educativos reales que se espera que sean analizados, comprendidos y transformados en beneficio de la comunidad afectada. Por lo tanto, la pedagogía crítica es contextualizada ya que observa las prácticas educativas 
en su contexto y analiza sus vínculos políticos y sociales. La pedagogía crítica encuentra su sustento en la teoría crítica (Borja, 2005).

\section{Aspectos metodológicos}

Con el propósito de llegar a un mayor nivel de profundidad en la comprensión del objeto de estudio se empleó una metodología sustentada principalmente en técnicas cualitativas para la recolección y análisis de la información.

En este sentido interesó, como lo menciona Taylor y Bogdan (1992), lograr un estrecho ajuste entre los datos provenientes del plan de estudios, de los programas cursos, de lo que realmente la gente dice y hace (opiniones, percepciones de docentes y estudiantes). Para mantener ese vínculo entre lo que se escribe y se dice que se hace, se parte de las propias palabras habladas y escritas, de los participantes en el estudio, lo que se contrasta con los lineamientos predefinidos en la instancia formadora de maestros.

\section{Fuentes de datos}

En el presente estudio se consideraron las siguientes fuentes de información:

Documentos: se revisa el plan de estudio de Bachillerato en Educación Primaria de la Sede de Occidente de la Universidad de Costa Rica; así como los programas de los cursos de Introducción a la Pedagogía, Principios de Curriculum, Didáctica General, Didácticas Específicas, Psicología Educativa, Práctica Docente, Tecnología Educativa, Investigación Educativa y Evaluación de los Aprendizajes.

Sujetos participantes: Los colaboradores de la investigación corresponden a siete profesores que atienden parte de los cursos indicados. Se consideraron, además doce estudiantes; los cuales cursan el tercero o cuarto año de carrera de Bachillerato en Educación Primaria y, en su mayoría han aprobado todos los cursos, o casi la totalidad de los cursos considerados en el estudio.

\section{Procedimientos}

Para aproximarse al objeto de estudio se aplicó un enfoque cualitativo, en el que se dio importancia a las opiniones y significados de los participantes acerca del objeto de investigación para llegar a un mayor nivel de profundidad en la comprensión y caracterización de las estrategias didácticas empleadas en los procesos de formación de docentes. 
Los momentos de la indagación hacen referencia al posicionamiento del objeto de estudio para su análisis, tanto en la estructura normativa que guía la función docente, como en la práctica académica concreta. Éstos se describen en cuatro fases: la primera correspondió al análisis de los lineamientos que orientan la formación de los docentes, la identificación de las metas por alcanzar en el plan de estudio, así como las estrategias didácticas previstas en el plan y programas de cursos. La segunda consistió en el estudio de las estrategias didácticas y las condiciones en las que éstas se desarrollan, en el aula, desde la perspectiva de los profesores. La tercera recoge la valoración de estas estrategias con base en la experiencia de los estudiantes y en la cuarta se contrasta, la información generada a partir de las categorías construidas para tal efecto.

Negociación: Dada la naturaleza del estudio y la necesaria inserción en la Sede de Occidente, se efectuaron procesos de negociación y de acercamiento por medio de la Coordinación del Plan de Estudio.

Recolección de la información: Para generar y sistematizar la información de documentos y de los participantes, se utilizaron las siguientes instrumentos de trabajo:

- Guías para el estudio del plan de estudio y programas de curso.

Para la revisión de estos documentos se hizo una lectura general preliminar y posteriormente una relectura $y$, con base en éstas, se construyen las guías que orientaron la atención y la búsqueda de los elementos necesarios para comprender la conceptualización del enfoque pedagógico y de las estrategias didácticas, así como para conocer los aspectos generales del mismo.

Basado en las guías respectivas se revisó: a). el plan de estudios para indagar sobre las características, estructura y enfoque pedagógico. b). los programas de cursos para identificar y describir las estrategias didácticas previstas en los programas.

En la confección de las guías para los talleres y los grupos focales, la estrategia didáctica fue considerada a partir de sus posibles componentes: métodos y técnicas didácticas, destrezas cognitivas, metacognitivas, actitudinales y motoras, relación teoría-práctica, relación con la realidad socio-educativa y cultural, empleo de la investigación, uso de materiales didácticos y las innovaciones educativas.

- Taller: Es una técnica que implica la reunión de un grupo de personas (10 a 30 participantes) para el estudio o discusión de un tema.

Esta técnica se aplicó con los profesores encargados de los cursos en estudio así como con los estudiantes, con el propósito de revisar y reflexionar acerca del desarrollo de la 
estrategia didáctica que se aplica en los procesos de enseñanza y aprendizaje en el aula universitaria.

Para efectuar estos talleres se prepararon dos instrumentos: una guía y una encuesta. La guía contiene instrucciones generales para ejecutar el taller y la encuesta preguntas abiertas que fue contestada, individualmente por los profesores participantes, con el propósito de revisar y reflexionar acerca de su práctica docente, ésta sirvió de base para analizar, de manera colectiva, la estrategia didáctica empleada en los cursos del área pedagógica.

Los talleres con profesores tuvieron dos etapas: un trabajo individual, que consistió en una auto-revisión de la estrategia didáctica empleada para el desarrollo del (de los) curso (s) a cargo y una plenaria para confrontar y analizar la estrategia didáctica aplicada en los cursos del área pedagógica. Para la preparación de este instrumento se consideraron los subproblemas $\mathrm{N}^{\circ} 2$ y 5 .

- Grupo Focal: esta técnica se empleó para conocer la opinión de los estudiantes con respecto al objeto de estudio. Al respecto se consideraron los subproblemas $\mathrm{N}^{\circ} 2,3,4$ y 5.

Para realizar el grupo focal se preparó una guía que consistió en una encuesta con preguntas abiertas, que los estudiantes participantes contestaron en forma individual, posteriormente se efectuó una discusión grupal con base en las respuestas de la encuesta y el apoyo de un moderador.

\section{Análisis de la información}

Para analizar los resultados se siguieron las siguientes acciones:

- Revisión de la información recopilada con el propósito de obtener categorías para analizar la información.

- Análisis de la información generada e incorporación del pensamiento de los actores sociales, de acuerdo con las fuentes de información (docentes y estudiantes).

- Discusión de resultados mediante la técnica de la triangulación con base en las categorías y según fuentes de información: análisis documental, consulta a los profesores y estudiantes.

- Socialización de resultados con los informantes a partir de sus propias creencias, significados y experiencias.

- Identificación de hallazgos significativos, mediante la confrontación de la discusión de resultados. 
Las categorías se construyen a partir del análisis de la información generada del problema y subproblemas planteados en el proyecto de investigación, así como de las conceptualizaciones elaboradas con base en la discusión de la literatura consultada y de las experiencias. Estas categorías son las siguientes:

- Mediación didáctica para el desarrollo de las lecciones

- Relación teórica-práctica

- El uso de los textos en las estrategias didácticas

- Condiciones en que se desarrollan las estrategias didácticas

- Innovaciones educativas

- Estrategias de evaluación

- Relación de las estrategias didácticas con los objetivos, los contenidos y la evaluación

- Relación de las estrategias didácticas con el enfoque pedagógico del plan de estudios.

\section{Análisis del Plan de Estudios}

\begin{tabular}{|l|l|}
\hline \multicolumn{2}{|c|}{ Descripción del Plan de Estudio } \\
\hline Institución & Universidad de Costa Rica. \\
\hline Título que otorga & Bachillerato en Educación Primaria. \\
\hline Salida Lateral & Profesorado en Educación Primaria. \\
\hline $\mathbf{N}^{\circ}$ de Créditos & $\begin{array}{l}\text { 135 en 8 semestres o ciclos, durante 4 años. Los créditos no incluyen } \\
\text { el Trabajo Comunal Universitario (300 horas). }\end{array}$ \\
\hline Año de Aprobación & 30 de marzo de 1977. \\
\hline Estructura & $\begin{array}{l}\text { Está organizado en 8 semestres, con una carga académica que } \\
\text { oscila entre 14 y 19 créditos por ciclo o semestre. }\end{array}$ \\
\hline Áreas & $\begin{array}{l}\text { Incluye humanidades, formación general y específica en las 4 áreas } \\
\text { fundamentales del curriculum escolar (Español, Estudios Sociales, } \\
\text { Matemática y Ciencias). } \\
\text { Ofrece 5 cursos optativos y 16 cursos con requisitos. }\end{array}$ \\
\hline $\begin{array}{l}\text { Relación en otras } \\
\text { universidades: }\end{array}$ & $\begin{array}{l}\text { Tiene relación con la Universidad Nacional Autónoma ya que el plan } \\
\text { en vigencia surge del que ejecutó esta Universidad en este recinto } \\
\text { universitario, aunque no está definida de manera explícita. }\end{array}$ \\
\hline
\end{tabular}

\section{Caracterización general del plan de estudio}

Al analizar el plan de estudios se logra identificar que se busca formar un profesional que cumpla con la misión que le exige el mundo de hoy, por lo que se aspira que el profesional de la educación:

- Sea capaz de satisfacer las demandas de un sistema educativo más funcional,

- Tenga una sólida preparación cultural, específica y didáctica, 
- Ostente una formación acorde con el desarrollo económico y social del país y con los adelantos de la ciencia, de la educación, del desarrollo de la tecnología y de la cultura universal.

- Posea una sólida formación humana, con habilidades profesionales basadas en las experiencias escolares para acercarlo a la realidad en la cual actuará.

De la descripción anterior se logra inferir que existe una intención por ejecutar un plan de formación de docentes con una visión de futuro; tratando de superar un enfoque pedagógico tradicional. Además, se evidencia una tendencia no muy definida hacia la pedagogía crítica porque se le da gran importancia a la realidad social y económica del país y en especial a la realidad escolar.

Por otra parte, aunque el plan de estudio no presenta estrategias metodológicas, se puede inferir de los objetivos, que éstas se centran en las experiencias del aula con un enfoque humanista y le dan gran relevancia a la investigación y a la práctica como un medio para construir el conocimiento. En la descripción de los cursos se describen proyectos de investigación, la relación que debe existir entre: la investigación y la práctica y la de la teoría con la práctica.

\section{Análisis de los programas de curso}

Los programas de los cursos estudiados por lo general presentan los siguientes elementos: descripción, objetivos generales y específicos (en algunos casos solamente se incluyen los generales), contenidos, metodología, evaluación, bibliografía y cronograma.

Con el análisis se pretende realizar una caracterización de los programas de los cursos desde la estrategia didáctica y su relación con los demás elementos curriculares. Para realizar este estudio se consideraron 16 programas de cursos y para la sistematización de los resultados se contó con una guía.

De este análisis se obtiene que:

1. Los métodos y las técnicas que los docentes utilizan con mayor frecuencia son: las exposiciones magistrales (por parte de los docentes y de los alumnos con temas asignados), prácticas (micro-enseñanzas utilizando diferentes estrategias didácticas) en el aula universitaria, demostraciones de técnicas didácticas por parte del docente y del alumnado, talleres con niños y padres de familia, comentarios de lecturas, trabajos en grupos e investigaciones especialmente bibliográficas.

2. Las principales destrezas cognitivas, metacognitivas y actitudinales que se pretenden desarrollar en los cursos son: 
- Cognitivas: conocimiento sobre los aspectos que repercuten en el proceso de enseñanza y aprendizaje, manejo de métodos, técnicas y procedimientos didácticos, construcción de recursos didácticos, conocimiento del fenómeno educativo y las concepciones en el contexto histórico, político, social y cultural, conocimiento de habilidades, actitudes sobre el crecimiento y desarrollo del niño, conocimiento de la teoría sobre la evaluación de los aprendizajes.

- Actitudinales: actitudes sobre los aspectos que repercuten en los procesos de enseñanza y aprendizaje, demostrar orden, responsabilidad ética, capacidad para trabajar con niños de I y II ciclos de la Educación General Básica,

- Metacognitivas: desarrollo de una actitud científica, estimulación del pensamiento reflexivo y crítico, resolución de problemas, capacidad para diseñar un proyecto de investigación, desarrollar destrezas relacionadas con la experimentación y criticar y valorar

3. Del análisis de los programas de los cursos se infiere que la relación del Plan de Estudios con la realidad de la escuela se pretende lograr por medio de prácticas en el desarrollo mismo de la clase como en aula escolar, observaciones de diferentes espacios educativos y por medio del planeamiento y ejecución de proyectos. Este último en menos grado.

4. Los cursos analizados, proponen una relación clara de la teoría con la práctica. Esta se pretende realizar mediante: observaciones de los procesos pedagógicos que se producen en el aula, talleres, micro-enseñanza en el aula (escuela, universidad), planeamiento de la labor diaria (práctica docente), realización de diagnósticos (aula, institución y comunidad), reuniones con padres de familia, la práctica docente, la aplicación de instrumentos de medición, ensayos por medio de micro-clases de la metodología estudiada.

5. Por otra parte, se observa, en los programas de cursos, que se propone la relación teoría práctica en sus contenidos y objetivos pero no se observa en las actividades descritas. Solamente en un curso se logró apreciar cómo implementar esa vinculación.

6. Al estudiar los programas de los cursos, se obtiene que de los catorce, trece no proponen el análisis de la práctica educativa. El único curso que sí lo menciona es el de práctica docente cuando analiza mediante las tutorías el trabajo que los practicantes ejecutan en las aulas escolares. Es probable que existan otros que lo realizan pero que en su planteamiento no se explicita. 
7. La investigación en la mayoría de los cursos la utilizan para: indagar sobre determinados contenidos de los cursos para luego presentarlos al grupo; por otra parte, la revisión de la literatura se utiliza como una "técnica útil para la enseñanza, la aplican a un tema... y se expone a la clase", para construir conocimiento, conocer la realidad en la cual el estudiante va a realizar su práctica docente. Es importante mencionar que la investigación es un componente fuerte en los programas de cursos que se analizaron.

8. Se identifica que solamente en dos, de los catorce cursos, se mantiene que se utilizan los medios de comunicación (videos) como un apoyo para el estudio de los diversos contenidos planteados en los programas.

9. Con respecto a la evaluación, en los programas de los cursos, se logra apreciar que incorporan como innovación educativa: la evaluación por procesos y proyectos, la utilización de la evaluación diagnóstica para determinar niveles de entrada y expectativas, la utilización de la evaluación diagnóstica, formativa y sumativa, la organización de la práctica docente: progresiva, intensiva, escalonada y la investigación como estrategia didáctica.

10. Los métodos y las técnicas que los docentes utilizan para desarrollar sus lecciones, según los programas de cursos, son: exposiciones, prácticas y demostraciones de técnicas didácticas, talleres, comentarios de lecturas, trabajos de grupos e investigaciones.

11. En los programas de curso existen diferentes relaciones entre la estrategia didáctica predominante y los elementos curriculares (contenidos, objetivos y evaluación):

- La relación interna entre los contenidos, objetivos y evaluación está presente; porque se enlistan los temas, las estrategias didácticas para su desarrollo y por último, se hace mención a los técnicas e instrumentos par su evaluación.

- La relación con los contenidos y objetivos: Cuando no existe relación con las estrategias de evaluación es porque se centra en la función sumativa, lo cual no es coherente con las estrategias didácticas propuestas.

12. Al revisar la relación entre la estrategia de didáctica general que se infiere del plan de estudios y la estrategia didáctica de los cursos se identifica que:

La gran mayoría de los cursos presentan una relación parcial: Esto porque en el plan de estudio, como se mencionó anteriormente, se pretende que las experiencias de aula y la investigación sean el insumo principal para la formación del futuro docente dentro de un enfoque humanista. Se dice que parcial porque en la mayoría de los cursos se considera solo la investigación bibliográfica. Solamente dos cursos hacen el 
intento por incorporar, en su desarrollo, la experiencia de los estudiantes en las aulas escolares.

13. Luego de razonar la información que se obtuvo de los programas de los cursos se logra determinar que existe influencia de:

- La pedagogía tradicional (diez cursos) porque se evidencia un desarrollo intelectual del estudiantado respecto al manejo de contenidos, métodos y técnicas; mediante la memorización de éstos no hay oportunidad para reflexionar sobre lo que se estudia (conocimiento estático), se promueve la incorporación del conocimiento al contexto social en forma abstracta, se da prioridad a métodos y técnicas que luego serán aplicadas, no se considera el contexto social como importante, se evalúa la reproducción de conocimiento.

- La pedagogía crítica: Este concepto pedagógico es llevado a la práctica en los cursos de Didáctica de las Ciencias y en los de Práctica Docente I y II ya que busca que el estudiante conozca su propia realidad por medio de la acción / reflexión, se intenta construir el conocimiento a partir de la realidad de aula, se utiliza la reflexión / acción como un medio para mejorar su práctica, se investiga, se aplica el trabajo grupal, la práctica pedagógica toma en consideración el contexto social, se utiliza la autorreflexión y se consideran requisitos para poder abordar los contenidos que se pretenden estudiar.

\section{Triangulación del Plan de Estudio y programas de curso}

Al establecer la relación teórica que existe entre el Plan de Estudio y los programas de cursos se logra identificar que:

- La tendencia didáctica que impulsa el Plan de estudios tiene vinculación con la tendencia de la pedagogía crítica con influencia de un enfoque humanista.

- La relación que se establece entre la estrategia metodológica que se promueve en el Plan de Estudio, con la de los programas de curso, se identifica como parcial; ya que el primero lo plantea por medio de la experiencia de aula y la investigación. Mientras que los cursos se hace por medio de prácticas, observaciones, demostraciones, investigaciones y proyectos en función del aula escolar y no a partir de esta.

- Con respecto a la formación del futuro docente y de acuerdo con los adelantos de la ciencia y la educación; existe un estancamiento, esto porque la mayoría de los cursos se ubican en un enfoque pedagógico tradicional; donde predomina la exposición magistral y la evaluación centrada en pruebas escritas. 
- No se está cumpliendo con la formación acorde con el desarrollo de la ciencia y la tecnología, ya que solamente dos de los catorce cursos analizados incorporan en alguna medida la tecnología.

- La mayoría de los cursos evidencian una estrategia didáctica fundamentada en la pedagogía tradicional (academicista); ya que su desarrollo se basa especialmente en exposiciones magistrales y lecturas, la evaluación es centrada en la medición de conocimientos y la evaluación sumativa.

- Existe una minoría de cursos que plantean una tendencia fundamentada en la pedagogía crítica.

- Se observó que tanto en el Plan de estudio como en la mayoría de los programas de curso se hace uso de la investigación (bibliográfica); lo cual indica que es el elemento más coherente entre estos componentes del Plan de Estudio y los programas de curso.

Luego de revisar los programas de cursos se evidencia la siguiente clasificación por tendencia pedagógica:

- Cursos que aplican estrategias con una fuerte vinculación con la pedagogía tradicional: Didáctica General, Bases Psicosociales de la Educación, Didáctica de la Matemática en Educación Primaria, Introducción a la Pedagogía II, Investigación Educativa, Crecimiento y Aprendizaje del Niño, Evaluación de los Aprendizajes en Educación Primaria y Preescolar, Lectoescritura y Diseño Curricular.

- Cursos con estrategias didácticas con una tendencia hacia la pedagogía crítica: Didáctica de las Ciencias y Práctica Supervisada I y II. Es conveniente aclarar que todavía existen elementos de la pedagogía tradicional en especial en la forma de evaluar.

\section{Talleres con profesores}

Se realiza un taller con el propósito de recoger información acerca de la estrategia didáctica que se utiliza en el desarrollo de los cursos del área pedagógica, desde la experiencia de los docentes. El taller estuvo estructurado en dos etapas. En la primera, cuando los participantes completan un instrumento, en la segunda se realiza una discusión guiada por parte de los investigadores, con el objetivo de clarificar y enriquecer la información que se obtuvo por medio del instrumento.

En el taller participan siete docentes que atienden catorce cursos del Plan de Estudios, estos cursos son: Didáctica de las Ciencias Generales, Desarrollo del niño, Volumen 5, Número 1, Año 2005 
Problemas de Ajuste y Aprendizaje, Salud Mental, Expresión Escrita para Educadores, Gramática para Primaria, Diseño Curricular, Didáctica General, Didáctica Específica, Introducción a la Pedagogía I, Introducción a la Pedagogía II, Práctica Supervisada I, Práctica Supervisada II, Administración Educativa y Didáctica de la Matemática en Educación Primaria.

Cabe destacar que de los anteriores cursos, los que fueron considerados para el estudio son: Didáctica General, Didáctica de las Ciencias Generales I, Práctica Supervisada I, Práctica Supervisada II, Desarrollo del Niño, Didáctica de la Matemática en Educación Primaria, Crecimiento y Aprendizaje del Niño, Lectoescritura, Introducción a la Pedagogía II. Es importante mencionar que se identificó un docente que ha impartido siete cursos del Plan de estudio en diferentes momentos.

\section{Hallazgos significativos}

Del análisis de la información obtenida del taller, se logra determinar que en la ejecución de los programas de cursos los docentes se caracterizan por presentar las lecciones con la siguiente estructura:

- Las lecciones por lo general, presentan la siguiente estructura: exposición de la teoría por parte de los docentes, luego se sigue con la ejemplificación de lo expuesto y se culmina con la ejecución de una práctica por parte de los estudiantes. Dentro del mismo proceso se promueve la participación de los estudiantes.

- Las principales actividades que los docentes realizan antes y después de las lecciones son:

Antes: Lecturas especializadas sobre los temas que se imparten en las lecciones, búsqueda y selección de información y fuentes bibliográficas, revisión de los programas de cursos, revisión de exámenes, planificar las lecciones a ejecutar, preparar algún material para las lecciones.

Después: Revisión de las tareas y prácticas asignadas, reflexión de lo que se logró y lo que queda pendiente, atención a los estudiantes en horas de oficina, lecturas sobre los temas a estudiar, revisión de exámenes y revisión de bibliografía.

- Los principales trabajos extraclases que los docentes asignan por lo general a los estudiantes, son: la preparación de materiales, lecturas de temas que se van a estudiar en clase, trabajos de investigación bibliográfica y participativa, entrevistas a docentes, elaboración de diagnóstico y observaciones del proceso de enseñanza y aprendizaje en instituciones educativas. 
- Los principales cambios que han realizado los docentes en el desarrollo de los cursos son: promover la participación de los estudiantes (incrementando más la práctica docente y la vivencia de aula), algunos contenidos, bibliografía (lecturas), metodologías (estilo de enseñar, evaluar) y la incorporación de ayuda audiovisual.

- Los recursos tecnológicos que los docentes utilizan en el desarrollo de sus cursos son: balanza electrónica y termómetros digital con fines demostrativos, informática para que los estudiantes realicen tareas o trabajos asignados y presentaciones en Power Point e Internet para búsqueda de información; la grabadora, el televisor, el retroproyector y multimedia también permite el desarrollo de las lecciones. Los docentes mencionan que se han sentido bien con la incorporación de la tecnología al proceso de enseñanza y aprendizaje, pero que están en proceso de adaptación; creen que necesitan capacitan en lo referente al uso y manejo de la tecnología aplicada a la educación.

- La evaluación que los docentes aplican en su mayoría son: pruebas escritas (cortas y parciales), proyectos de investigación, exposiciones, trabajos grupales e individuales, e indicadores como la participación del estudiante en el desarrollo de los cursos y la autoevaluación al final del curso.

- Con respecto a la planta física, los docentes mencionan que las condiciones son adecuadas; en relación con la biblioteca consideran que hay un buen servicio, que se cuenta con medios tecnológicos que no están en estado óptimo y son de difícil acceso; por último opinan que hace falta material didáctico.

- Los docentes creen que el enfoque pedagógico del plan de estudio tiene tres enfoques:

- Conductista-academicista; porque el plan está muy cargado de contenido.

- Humanista; porque se busca una formación integral del futuro docente.

- Constructivismo; porque se hacen esfuerzos por construir el conocimiento por medio de los procesos de enseñanza activos y participativos.

- Los docentes consideran que los cursos contribuyen en la formación de los estudiantes en el área cognitiva, valorativa, afectiva y ética porque se promueve una formación integral y reflexiva; un carácter crítico, perseverante, experimental, humanista, para que puedan enfrentarse a la labor de aula y así mejorar la calidad de la formación del estudiante. 


\section{Grupo focal con alumnos}

Esta actividad con estudiantes, se planeó con el propósito de recoger información y apreciaciones acerca de las estrategias didácticas utilizadas en el desarrollo de los cursos del área pedagógica, del Plan de Estudios de Educación Primaria.

El trabajo se organizó en dos etapas. En la primera el estudiante completó un instrumento y en la segunda participó en una discusión guiada por parte del investigador con el objetivo de clarificar y enriquecer la información que se obtuvo por medio del instrumento.

\section{Hallazgos significativos}

- Los estudiantes consideran que los métodos predominantes en los procesos de formación son los tradicionales y en especial las exposiciones.

- Los principales trabajos extraclase que se utilizan son las lecturas, observaciones y trabajos de investigación.

- Se considera que la tecnología se usa muy poco. Se aplica en el curso de tecnología educativa y preparación de material educativo. El principal cambio se centra en el uso de la Internet para realizar búsquedas; así como la informática para realizar presentaciones de Power Point.

- Se identifica que la evaluación más utilizada es la sumativa y en especial la aplicación de pruebas escritas.

- Los alumnos consideran que el equipo audiovisual y material bibliográfico con que se cuenta está disponible, pero no se utilizan correctamente por parte de los docentes, además ambos deben ser actualizados.

- Al referirse a las instalaciones opinan que las aulas son pequeñas para los grupos con más de treinta personas, aunque la planta física la valoran como excelente.

- En lo que respecta enfoque pedagógico del Plan de estudios, se considera que permite formarse una visión más clara y práctica de lo que es la labor docente desde enfoques de estilo conductista, academicistas principalmente; también se menciona la presencia del humanismo y una tendencia hacia el constructivismo. Se reconoce que se hacen esfuerzos por construir el conocimiento y por realizar procesos activos y participativos, pero se evidencia desconocimiento por parte del docente respecto a estos últimos enfoques.

- Se identificó que los estudiantes conocen el Plan de estudio; en especial lo relacionado al perfil, objetivos y propósitos. 
- La contribución del Plan de estudio a la formación la consideran como muy amplia y enriquecedora; además valoran que la calidad de formación es muy buena.

- Con respecto al Plan de estudio lo consideran muy completo ya que abarca aspectos como personalidad, problemas, didácticas específicas, investigación, tecnología y preparación de material didáctico entre otros.

\section{Discusión Final}

En este estudio se realizó un recorrido que permite comprender y establecer vínculos entre el Plan de estudio, los programas de cursos y los criterios de los docentes y los alumnos desde la práctica de aula. Estos resultados se presentan a partir de las categorías de análisis y subproblemas planteados anteriormente.

\section{Subproblemas uno y dos:}

- El Plan de estudio y programas de cursos, en general, poseen una estructura muy similar respecto a su: descripción, objetivos, contenidos, criterios de evaluación, cronograma y bibliografía. La descripción de la estrategia metodológica como tal no se presenta en forma explícita sino que se debe inferir de la fundamentación teórica, del enfoque pedagógico del plan, de las actividades y criterios de evaluación.

- Las tendencias pedagógicas que se identifican, en su orden, son la tradicional y cierta tendencia hacia la constructivista. Hay un predominio de destrezas cognoscitivas, con pocas excepciones aparecen las de la Metacognición y actitudinales; no se hace referencia a las destrezas psicomotoras. Puede afirmarse que los lineamientos y orientaciones teórico-prácticas se explicitan en el plan de estudio, pero se diluyen o no están presentes en los programas de los cursos.

- En el área de la evaluación de los aprendizajes, en el plan de estudio no se especifica claramente, y en los programas de cursos se tiende a combinar tipos y funciones de la evaluación, ésta enfatiza la medición y el carácter sumativo. Además los procesos grupales e individuales de producción, creación y aplicación no son valorados por medio de procedimientos de coevaluación y autoevaluación.

- El Plan de estudio presenta cierta coherencia con las recomendaciones que establecen organizaciones internacionales como la UNESCO y la CECC respecto a la formación de formadores en el nivel inicial, en cuanto a incorporar la investigación como método de enseñanza. 
- Las estrategias didácticas aplicadas se presentan como una serie de técnicas empleadas en el desarrollo de los diferentes cursos; dentro de ellas prevalecen: exposiciones de los docentes y estudiantes en forma individual y grupal con base en la lectura e investigación bibliográfica, observaciones, prácticas en escuelas de Educación Primaria, ejecución de propuestas pedagógicas (prácticas).

- Los programas de curso no aportan suficientes elementos para caracterizar una estrategia didáctica; lo que se encuentra es un listado de actividades registradas en los respectivos cronogramas para desarrollar una serie de contenidos y alcanzar unos objetivos propuestos.

- El plan de estudio ofrece lineamientos generales que orientan la elaboración de los programas de cursos, al privilegiar determinados enfoques pedagógicos con fundamento en el humanismo como enfoque curricular. Señala además la necesidad de incorporar los avances científicos y tecnológicos así como el ofrecer respuestas a las demandas del contexto social. Sin embargo ello no es evidente en el programa de los curso.

- Los programas de los cursos indican el empleo de estrategias centradas en el docente y en el desarrollo de contenidos, lo que no es coherente con el plan de estudio.

\section{Subproblemas dos y cuatro son:}

- Se logró apreciar un evidente distanciamiento entre el discurso docente y su práctica. Esta falta de consistencia no es reconocida por las opiniones y creencias de los profesores, sino por las apreciaciones de los estudiantes quienes señalan actuaciones contradictorias entre el discurso y la práctica. El enfoque predominante es el centrado en el docente, en los contenidos de las asignaturas, en los exámenes y las excesivas lecturas.

- La práctica pedagógica en el aula universitaria, se diversifica y se enriquece con el empleo de diversas técnicas y recursos didácticos por parte de los estudiantes, incluyendo de manera especial los que corresponden a las actividades extraclase.

- La evaluación se utiliza como un medio de control del rendimiento del estudiante en los procesos de enseñanza y aprendizaje. Con la excepción de aquellos cursos que toman como referencia la práctica docente. En el plan de estudio se observa que la evaluación es empleada como un medio para mejorar los procesos de enseñanza y aprendizaje; mientras que en los programas de los cursos se aprecia únicamente la función sumativa con un carácter unidireccional. 
- Con respecto a la incorporación del contexto socioeducativo en los procesos de formación de docentes, en el plan de estudio se identifica la necesidad de considerarlo como punto de partida; mientras que en los programas de curso se busca establecer vinculación con éste, por medio de los trabajos extraclases (observaciones, prácticas e investigaciones).

- Los programas de curso, no reflejan la riqueza pedagógica que se puede generar a partir del trabajo en grupos, de las prácticas y observaciones en el aula escolar, los productos de los ejercicios investigativos y las reflexiones surgidas de las lecturas.

- La lectura como complemento a la exposición o la discusión constituye la técnica didáctica de mayor uso. El abordaje didáctico que hace el docente dificulta al estudiante hacer la relación teórico-práctica.

- Los curso de Práctica Docente, Ciencias y Didáctica de la Lectoescritura obtienen una valoración positiva. Esta se podría atribuir a que estos cursos logran acercarse a la realidad de aula que el estudiante va a enfrentar como futuro formador.

- El espacio físico como un componente importante en donde se desarrollan los procesos de formación no es considerada una limitación.

En relación con el subproblema cinco el cual hace referencia a la incorporación de estrategias didácticas innovadoras se encuentra lo siguiente:

- La incorporación de la tecnología informática en el desarrollo de los cursos es mínima. Los programas no la consignan y los estudiantes señalan que son pocas las posibilidades de acceso a los equipos.

- Los docentes aducen falta de capacitación y de disponibilidad institucional del equipo. El uso principal que se hace es búsquedas de información en Internet y presentaciones en Power Point y elaboración de material didáctico.

- La tecnología se utiliza como un medio para hacer más atractivo el proceso de enseñanza y aprendizaje; lo cual no constituye un cambio de paradigma.

- Se identifican otros elementos que los estudiantes consideran innovadores en el sentido que rompen con la rutina o monotonía de la clase "magistral", de la lectura o trabajo en grupo al darles un mayor protagonismo. Se trata de los talleres, giras e investigaciones.

- Se aprecia como novedoso la incorporación de la investigación como estrategia didáctica.

- Por último es importante destacar, que a partir de los resultados obtenidos se hace necesario considerar la historicidad del plan de estudio, para comprender su estado y 
justificar la necesidad de incorporar procesos autoreflexivos en la formulación y ejecución del plan de formación de docentes.

Para obtener información del análisis de cada plan de estudios, se recomienda consultar el informe correspondiente (Hernández, Montenegro, Francis y Gonzaga, 2002).

\section{Referencias}

Abarca, S. (1995). Psicología de la motivación. San José, Costa Rica: EUNED.

Aguilar A., Hugo; Goldoni R., Catalina. (1999). Acerca del Pensar y el Hacer: una reflexión crítica desde la práctica educativa. En UMBRAL, 10, 10 -75.

American Psichological Association. (1999). Manual de estilo de publicaciones de la American Psichological Association. México: Editorial El Manual Moderno.

Borja, Mónica. (2005). La pedagogía crítica y la contextualización de la enseñanza. En Revista Eureka. No $\quad \mathrm{N}^{\circ}$. Universidad del Norte. España. http://www.uninorte.edu.co/publicaciones/eureka/edicion03/monica borja.html

Contreras Montes de Oca, lleana. (1994). El rol del docente como objeto de estudio. Revista Educación, 18(1), 63-71.

Coordinación Educativa y Cultural Centroamericana (CECC) Secretaria General. (2000). Ayuda Memoria Taller Latinoamericano para definir el perfil marco del docente de la Educación Primaria o Básica. San José, Costa Rica: CECC.

Coordinación Educativa y Cultural Centroamericana (CECC) Secretaria General. (2000). Perfil Marco Centroamericano del docente de la Educación Primaria o Básica. San José, Costa Rica: CECC.

Coordinación Educativa y Cultura Centroamericana (CECC). (1996). Memoria Taller regional de la Formación de Docentes de Educación Primaria o Básica. San José, Costa Rica: CECC.

Galindo Cáceres, Jesús. (1998). Técnicas de investigación en sociedad cultura y comunicación. México: Addison Wesley Longman.

Gallego Badillo. Rómulo. (1992). Saber pedagógico: Una Visión Alternativa. Santa Fe de Bogotá: Cooperativa Editorial Magisterio.

Hernández R., Ana Cecilia y Solano A., José. (1995). La formación docente inicial para la Educación General Básica: el caso de Costa Rica. San José, Costa Rica: Facultad de Educación. Universidad de Costa Rica.

Hernández R., Ana Cecilia. (1999). Formación pedagógica de los docentes universitarios. Revista Educación, 23(Especial), pp. 91-104. 
Hernández R., Ana Cecilia. (2000). Estrategias innovadoras para la formación docente. Conferencia presentada en el Taller Regional para la definición del perfil marco del docente de la Educación Primaria o Básica. San José, Costa Rica: CECC.

Hernández R., Ana Cecilia; Montenegro H., Manuel; Francis S., Susan y Gonzaga M., Wilfredo. (2002). Estrategias didácticas empleadas en la enseñanza del área pedagógica en los planes de formación inicial de docentes para la Educación primaria en las universidades estatales costarricenses. Informe Final Investigación. San José, Costa Rica: CECC.

Méndez Sánchez, Zayra. (2002). Aprendizaje y cognición. San José, Costa Rica: EUNED.

Pansza G., Margarita; Pérez J., Esther; Morán O., Porfirio. (1997). Fundamentación de la Didáctica. Vol. I. México: Ediciones Génica.

Programa de Naciones Unidas para el desarrollo (PNUD). (1999). Los retos educativos del futuro. Estado de la Educación en América Latina y el Caribe. Costa Rica: Guilá Imprenta Litográfica.

Ruiz O., José y Ispizua, María. (1989). La descodificación de la vida cotidiana. Métodos de investigación cualitativa. Bilbao: Universidad de Deusto.

Saavedra, M. (1998). Formación de docentes reflexivos transformativos. Revista de Tecnología Educativa, 13(2), 191-211.

Stebhouse, L. (1991). Investigación y desarrollo del currículo. $3^{\circ}$ ed. Madrid: Editores Morata.

Taylor, S. J. y Bogdan, R. (1992). Introducción a los métodos cualitativos. La búsqueda de significados. Buenos Aires: Piados.

UNESCO y Universidad Pedagógica Nacional Libertador. (1996). Seminario Regional para La formación docente inicial para la Educación Básica. Santiago, Chile: UNESCO. 